rechallenge with omeprazole resulted in a much greater deterioration of renal function than was apparent at the time of original exposure, suggesting a process of sensitisation.

Our report demonstrates the need to con-

1 Assouad M, Vicks SL, Pokroy MV, Willcourt RJ. Recurrent acute interstitial nephritis on rechallenge with omeprazole (letter). Lancet 1994;344:549.

2 Christensen PB, Albertsen KEP, Jensen P. Renal failure after omeprazole. Lancet 1993;341:55.

Ruffenach SJ, Siskind MS, Lien Y-HH. Acute interstitial nephritis due to omeprazole. Am F Med 1992;93:472-3.

4 Kuiper JJ. Omeprazole-induced acute interstitial nephritis. Am F Med 1993;95:248.

5 Jones B, Hewson E, Price A. Acute interstitial nephritis due to omeprazole (letter). Lancet 1994;344:1017-8.

6 Lewis CR, Somerville C, Agar JW. Omeprazole induced acute interstitial nephritis (letter). Aust NZ $\mathcal{F}$ Med 1994;24: 578. sider drugs as a cause of unexplained fever. The serendipitous rechallenge with the suspected agent confirms the diagnosis of omeprazoleinduced pyrexia with bone marrow hypoplasia and interstitial nephritis.

7 Singer S, Parry RG, Deodhar HA, Barnes JN. Acute interstitial nephritis, omeprazole and antineutrophil cytoplasmic antibodies (letter). Clin Nephrol 1994;42:280.

8 Ottervanger JP, Stricker BHC, Kappelle JW, Claas FHJ Omeprazole-associated agranulocytosis. Eur $f$ Haematol 1995;54:279-80.

9 Marks DR, Joy JM, Bonheim NA. Haemolytic anaemia associated with the use of omeprazole. Am $\mathcal{F}$ Gastroenterol $1991 ; 86: 217-8$

\title{
Oesophageal perforation: a dangerous but potentially curable condition
}

\author{
S Mahmoud, R Prudham, G Sidra, S Solomon
}

\section{Summary \\ A series of three cases of oesophageal per- foration are described. All three presented differently and the $X$-ray findings were different in the three patients.}

Keywords: oesophageal perforation; Boerhaave syndrome

Oesophageal perforation is a potentially fatal condition. It should be recognised and treated appropriately to prevent death. We report three cases, each of which presented differently, followed by a review of the subject.

\section{Case reports}

Case 1

A 73-year-old woman was admitted as an emergency at her general practitioner's request with an 8-hour history of persistent vomiting. There was no previous history of note; this was her first admission to hospital. Physical examination revealed tachycardia at a rate of 122 beats/min and blood pressure of 185/90 $\mathrm{mmHg}$. She was noted to be pale and dehydrated although her routine bloods were within normal limits. Abdominal examination demonstrated minimal epigastric tenderness with no other abnormality.

Plain abdominal X-ray was normal and the vomiting settled overnight. She was reviewed the following morning and by that time had developed subcutaneous emphysema. Review of her chest X-ray demonstrated mediastinal emphysema.

The patient was kept nil by mouth and the clinical suspicion was of a high oesophageal tear. Gastrografin swallow was performed but

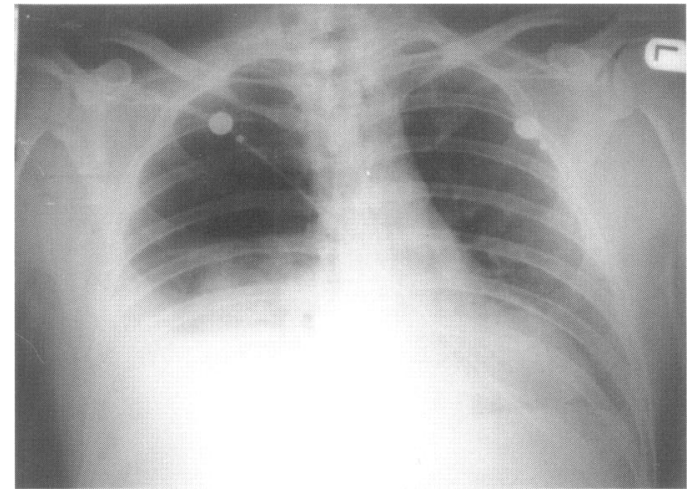

Figure Chest X-ray

no tear could be identified. In view of this, fluids and then solids were re-introduced by mouth successfully and the patient suffered no further problems. She was then discharged home after satisfactory review by the ENT surgeons. An alternative diagnosis, which should be considered, is that she had suffered from a rupture of a pulmonary bleb. However, since she was symptomatic on admission with normal X-ray, the diagnosis of oesophageal tear is, in our opinion, more likely.

\section{Case 2}

A 50-year-old man presented to the Accident and Emergency department with chest pain and sudden onset dyspnoea. Four hours earlier he had awoken with nausea and he had proceeded to vomit violently with subsequent retching. He denied having drunk excessive alcohol or having consumed a particularly large meal. There was no history of haematemesis or coffee-ground vomitus. 
There was no medical history of note; the patient was taking no medications. Physical examination revealed a distressed patient with tachypnoea of 24 breaths $/ \mathrm{min}$. Blood pressure was $130 / 75 \mathrm{mmHg}$, pulse 100 beats/min and regular. Auscultation demonstrated a loud second heart sound. The trachea was noted to be deviated to the left. Percussion note was hyperresonant throughout the right side except in the base where dullness was noted. Abdominal examination simply revealed epigastric tenderness with no signs of peritonism. Chest X-ray confirmed the clinical diagnosis of a right-sided tension pneumothorax with mediastinal shift to the left (figure). There was also a right-sided effusion on the X-ray. ECG demonstrated sinus tachycardia with evidence of right ventricular strain. Pulse oximetry demonstrated an oxygen saturation of $81 \%$ on high flow oxygen with a reservoir bag.

An 18 French gauge chest drain was inserted in the right posterior axillary line via the 6 th and 7 th intercostal space. Altered blood was seen to drain freely. The clinical diagnosis was oesophageal rupture with secondary tension pneumothorax. The patient then had a watersoluble contrast swallow examination, which showed barium leaking freely into the drain and the right pleural cavity. The patient was therefore transferred to a cardiothoracic centre for consideration for surgical repair. At thoracotomy a longitudinal full thickness tear was revealed at the junction of the middle and lower third of the oesophagus. Fluid was also identified within the pleural cavity. The laceration was closed with a double layer of $4 / 0$ Prolene, the suture line covered by a pedicle of intercostal muscle. After 6 days of parenteral nutrition a further barium swallow showed no leakage and he was successfully restarted on a light diet. He went on to make a good recovery.

\section{Case 3}

A 73-year-old woman was admitted with a 1-day history of chest pain radiating to her back. The pain was partly relieved by glyceryl trinitrate (GTN) spray. The patient was known to have ischaemic heart disease and chronic obstructive airway disease. She was taking salbutamol inhaler, ipratropium inhaler, becotide inhaler, isosorbide mononitrate, nifedipine SR and GTN spray.

Physical examination on admission showed the patient was generally ill but with no other specific findings. Serial electrocardiograms did not show any evidence of acute myocardial infarction. Cardiac enzymes were within normal. Full blood count showed high white cell count with $83 \%$ neutophilia. The patient continued

\footnotetext{
1 Reeder LB, Defillipei VJ, Fergusson MK. Current results of therapy for oesophageal perforation. $A m \quad \mathcal{F}$ Surg 1995;196:615-7.

2 Ghahremani GG. Radiologic evaluation of suspected gastrointestinal perforations. Clin North Am 1993;31:1219-
}

\section{Causes of oesophageal perforations}

- iatrogenic: instrumental, postoperative

- swallowed foreign bodies

- external trauma, usually penetrating

- corrosive ingestion

- spontaneous: intramural haematoma,

Mallory-Weiss syndrome, Boerhaave syndrome

- progressive disease: peptic ulceration, hiatus hernia, tumours

to deteriorate and developed abdominal pain and diarrhoea. Repeat clinical examination revealed generalised abdominal tenderness and rigidity. Chest and abdominal X-ray and ultrasound of the abdomen were normal. A diagnosis of acute abdomen was made and the patient was referred to the surgeons. The patient was deemed to be too frail for surgical intervention and treated conservatively. Post-mortem examination revealed that the cause of death was a perforated oesophagus secondary to an oesophageal ulcer. There was no evidence of malignancy. The perforated ulcer was approximately $2 \mathrm{~cm}$ in diameter within the middle third of the oesophagus. It perforated anteriorly into the posterior mediastinum causing mediastinitis.

\section{Discussion}

The terms oesophageal perforation and rupture are used synonymously with respect to oesophageal injuries. The causes of oesophageal perforation are shown in the box.

Our first case had incomplete perforation. The second and the third cases had Boerhaave syndrome. Oesophageal perforations are classified into early (acute) and late (chronic). The former is associated with good prognosis and the latter with a bad prognosis. ${ }^{1}$ The clinical features of the condition include pain, tachycardia and haematemesis. Surgical emphysema is observed in $60 \%$ of cervical and $30 \%$ of mid-oesophageal injuries. In thoracic injuries respiratory distress is common, as in case 2 . The diagnosis is confirmed with chest X-ray which shows surgical emphysema and may show hydropneumothorax, as also demonstrated in case 2. A normal X-ray does not exclude the diagnosis, ${ }^{2}$ as shown in case 3 . Gastrografin studies of the oesophagus can show the site of the perforation. The treatment is surgical unless the injury is incomplete when conservative treatment with antibiotics and parenteral nutrition is sufficient. ${ }^{3}$

\footnotetext{
3 Cuschierie A, Giles GR, Moossa AR. Essential surgical practice. Butterworth-Heinemann Ltd, 1992; pp 930-2.
} 